\title{
Histiocytose Langerhansienne à localisation multiple. À propos d'un cas
}

\section{RÉSUMÉ}

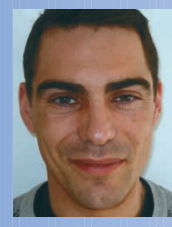

Fabrice CLIPET

Assistant Hospitalo-Universitaire,

Service de chirurgie buccale,

C.H.U. de Rennes,

Faculté d'Odontologie,

Université de Rennes I,

2, avenue du Professeur Léon Bernard,

Bâtiment 15 ,

35043 Rennes Cedex.

\section{Nora ALNO}

Interne en Odontologie,

Service de chirurgie buccale,

C.H.U. de Rennes,

Faculté d'Odontologie,

Université de Rennes I.

\section{Gérard LANCIEN}

Professeur des Universités,

Praticien Hospitalier,

Département d'Anatomie et Cytologie

Pathologiques,

C.H.U. de Rennes.

\section{Gilbert DE MELLO}

Professeur des Universités,

Praticien Hospitalier,

Service de chirurgie buccale,

C.H.U. de Rennes,

Faculté d'Odontologie,

Université de Rennes I.

L'histiocytose Langerhansienne est une maladie rare caractérisée au niveau histologique par la présence de foyers granulomateux contenant de nombreuses cellules langerhansiennes.

Le cas présenté concerne une histiocytose Langerhansienne à localisation multiple. À la suite du bilan dentaire, on constate la présence de lésions cliniques se traduisant au niveau radiologique par des images radioclaires. L'analyse anatomopathologique positive aux CD1a confirme l'origine langerhansienne de ces lésions.

La prise en charge des lésions alvéolo-dentaires consiste en un curetage chirurgical en première intention. Lorsqu'une lésion récidive, diverses stratégies peuvent alors être adoptées : la radiothérapie à faible dose, la chimiothérapie, les traitements anti-inflammatoires. Dans tous les cas un suivi très régulier doit être instauré.

\author{
histiocytose \\ Langerhansienne \\ - maxillaire \\ - mandibule \\ curetage
}




\section{Introduction}

$>$

L'Histiocytose Langerhansienne est une

maladie dont on estime la prévalence à 4 à 5,4 pour un million[21, 23, 28]. Elle se qualifie selon le rapport émis en 1987 par la Société d'Histiocyte[1] par des foyers composés d'une prolifération granulomateuse de cellules langerhansiennes, d'histiocytes normaux, de lymphocytes et de granulocytes éosinophiles.

L'étiopathogénie n'a pas encore été précisément déterminée[8]. La nomenclature actuelle s'appuie donc sur la clinique et distingue les formes localisées (le granulome éosinophile), les formes disséminées aiguës (la maladie de Letterer-Siwe), et les formes disséminées chroniques (la maladie de Hand-Schüller-Christian).

Le tableau clinique rencontré dans ces pathologies dépend de la localisation du foyer initial, du nombre d'organes atteints et de la répercussion de la pathologie sur la fonction de ces organes.

Dans les atteintes osseuses alvéolaires et gingivales, les premiers signes de la pathologie sont analogues à ceux d'une parodontopathie. Les gencives sont hypertrophiques, souples, de couleur rouge vif. L'os alvéolaire présente des foyers de résorption notamment au niveau des espaces inter dentaires[8]. Dans certains cas, la lyse osseuse peut s'étendre jusqu'au niveau apical simulant une alvéolyse terminale[18]. Radiologi-

\section{Cas clinique}

En août 2006, suite à la découverte d'une localisation vertébrale d'une histiocytose Langerhansienne, une patiente de 47 ans est adressée dans notre service de Pathologie et de Chirur- quement, les dents semblent alors "flotter»[4] dans l'alvéole où il ne reste plus qu'un tissu granulomateux comme support dentaire[4, 7].

Dans les histiocytoses qui s'étendent au niveau de la base du crâne, les lyses osseuses peuvent toucher la selle turcique et aboutir à des perturbations du système hypothalamo-hypophysaire qui simulent un diabète insipide[13, 25]. Le déficit en hormone de croissance et l'hypogonadisme sont quant à eux des symptômes beaucoup moins fréquents. Dans les localisations mastoïdes et temporales, on peut observer des inflammations du méat acoustique externe, des otites ou des mastoïdites.

Les localisations vertébrales aboutissent à des raréfactions osseuses du corps vertébral et entraînent une fragilisation nécessitant le port d'un corset et I'alitement temporaire du patient qui ne sera levé que 6 semaines après réalisation d'une arthrodèse.

En raison de ces grandes variations cliniques, seul l'examen histopathologique permet de poser le diagnostic positif d'histiocytose[11]. De plus, pour les localisations gingivales, une immunohistochimie utilisant un anticorps pour CD1a complète I'analyse[18]. Elle permet de distinguer les foyers gingivaux d'histiocytose des modifications cellulaires inflammatoires des maladies parodontales.

gie Buccale par le service de Rhumatologie pour recherche de foyer infectieux bucco-dentaire. 


\section{Aspects cliniques}

\section{> Antécédents personnels}

Cette patiente a présenté un granulome éosinophile à localisation crânienne. Ce foyer a été cureté et des plaques d'ostéosynthèse ont été posées dans le service de neurochirurgie, en juin 2006. Le suivi ne montre pas actuellement de récidive du granulome éosinophile.

\section{> Anamnèse}

En août 2006, Mme X est adressée dans le service de rhumatologie pour une lombalgie de rythme nocturne accompagnée de céphalée en casque postérieur sans cervicalgie. II n'y a pas de trouble de la statique ni de raideur cervicale. Après avoir réalisé un diagnostic d'exclusion, notamment d'une pelvispondylite rhumatismale, des examens radiologiques tomodensitométriques et IRM mettent en évidence des lésions ostéolytiques de L4, L5 et de l'aile iliaque gauche.

Les biopsies osseuses de ces trois foyers montrent au sein d'un tissu conjonctif oedématié des plages cellulaires denses constituées de nombreux leucocytes polynucléaires éosinophiles auxquels se mêlent des éléments de caractère histiocytaire à cytoplasme assez étendu, modérément basophile et assez souvent surchargé de cristaux de Charcot Leyden. Ces altérations vont dans le sens d'une histiocytose langerhansienne de forme osseuse pluri focale sans atteinte vasculaire[6].

La réalisation d'une arthrodèse lombaire et la mise en place d'un traitement par corticoïdes[15] sont alors décidées.

Au préalable, la patiente nous est adressée pour un bilan de recherche de foyer infectieux bucco-dentaire.

\section{Examen bucco-dentaire}

À l'entretien, la patiente ne se plaint d'aucune douleur. Elle nous dit être en cours de soins pour une maladie parodontale.

\section{- Exo buccal}

La palpation des territoires ganglionnaires ne met pas en évidence d'adénopathie cervicofaciale.

L'examen des téguments est normal.

Il n'y a pas de perturbation fonctionnelle de l'articulation temporo-mandibulaire.

\section{- Endobuccal}

- Muqueux :

II n'y a pas de plaque ni de tartre. Néanmoins, même s'il n'y a pas d'inflammation généralisée, on peut voir des zones muqueuses hémorragiques souples et rouge vif au niveau de 28 , 45,46 et 48 (fig. 1).

Le sondage révèle la présence de poches parodontales:

- de $12 \mathrm{~mm}$ entre 45 et 46 ;

- de $10 \mathrm{~mm}$ entre 47 et 48 ;

- de 10 mm en mésial de 28 .

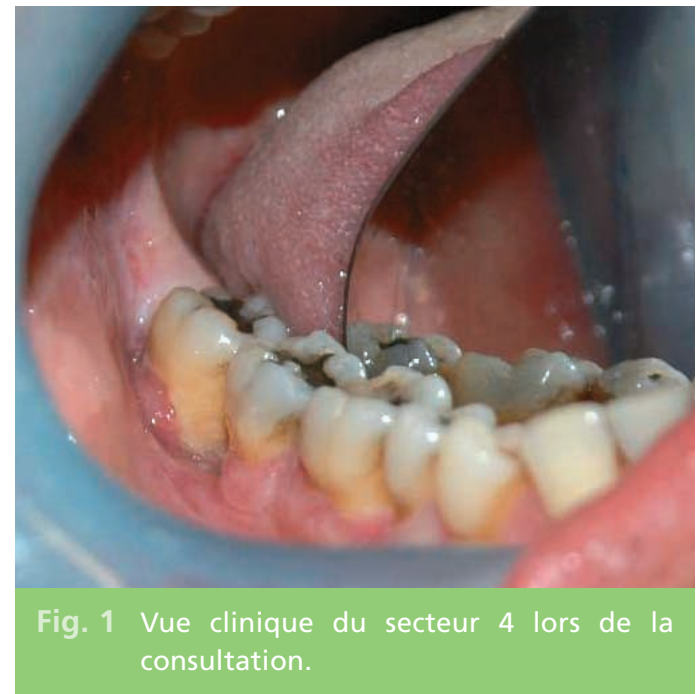


- Dentaire :

La formule dentaire est incomplète suite à des avulsions de 38 et 37 réalisées en décembre 2006. La patiente nous signale que l'indication d'extraction était parodontale. Nous pouvons cependant raisonnablement penser qu'il s'agissait d'une première localisation buccale de I'histiocytose.

Les dents suivantes, 28,45 et 48 présentent une mobilité 3 suivant la classification ARPA. Elles répondent aux tests de vitalité.

La 46 n'est pas mobile et ne présente aucune sensibilité.

- Imagerie :

Le panoramique confirme la présence de lyse osseuse (fig. 2).

- En mésial de 28 ;

- en inter proximal de 45, 46 (fig. 3) ;
- en mésial de 48 (fig. 3).

II n'y a pas d'autre image radiologique pathologique.

\section{Diagnostic}

En raison du contexte médical et du caractère localisé des lésions parodontales, le diagnostic d'histiocytose à localisation buccale est évoqué[9, 12].

\section{> Plan de traitement}

Il consiste à éliminer tous les foyers potentiels d'histiocytose[2,14]. Les dents 28,45 et 48 seront extraites sous anesthésie locale en un temps opératoire avec exérèse du tissu granulomateux (fig. 4) et curetage osseux appuyé. Les tissus d'exérèse de chaque site sont placés

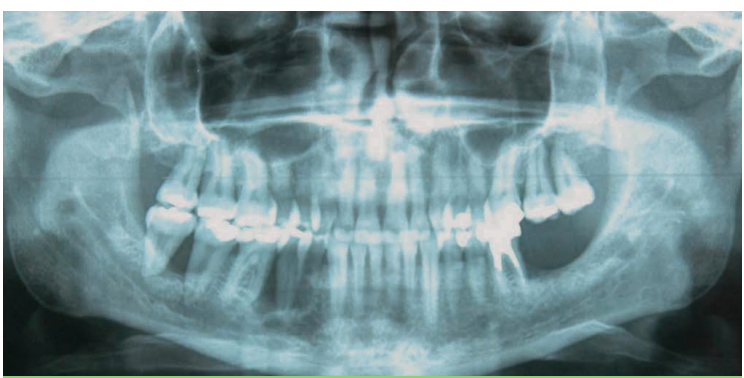

Fig. 2 Radiographie panoramique

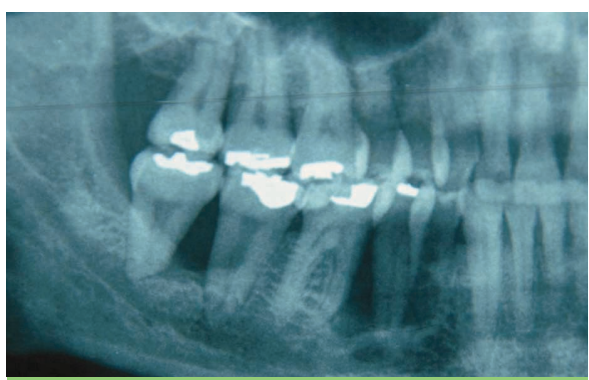

Fig. 3 Image radiographique des pertes de substance osseuse dans le secteur 4.

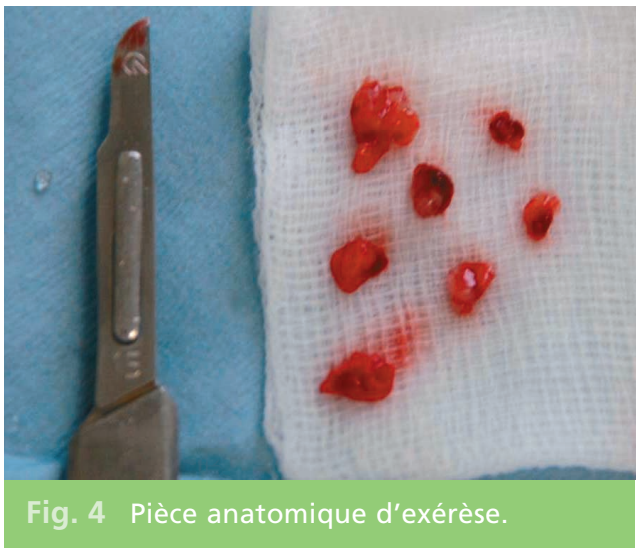




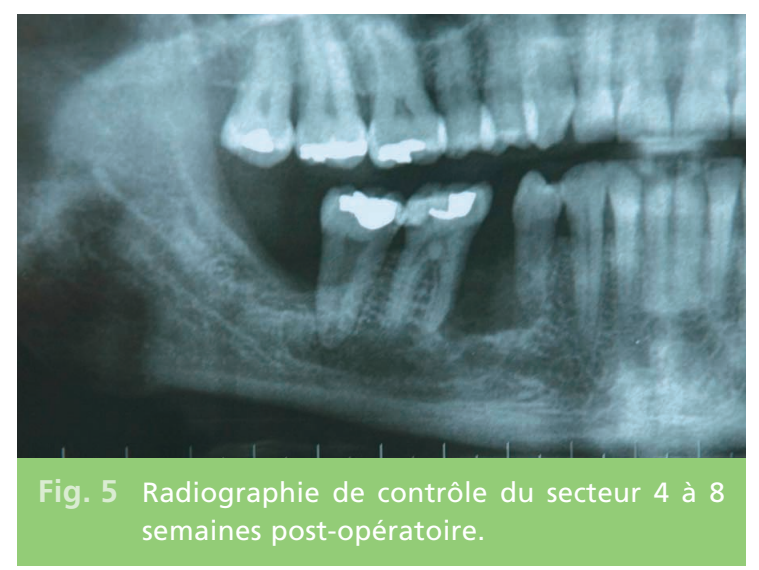

dans des flacons d'analyse distincts contenant un liquide de Bouin. Puis ils sont envoyés au laboratoire d'anatomo-pathologie pour analyse histologique et immuno-histochimique.

En raison de l'absence de mobilité et bien qu'elles soient contiguës aux sites d'alvéolyse 46,47 et 27 sont conservées.

\section{> Résultats anatomopathologiques}

Les différents prélèvements sont d'aspect microscopique identique. Le chorion est le siège d'un infiltrat inflammatoire composé de polynucléaires, de lymphocytes, de polynucléaires éosinophiles et surtout de grandes cellules à noyau réniforme correspondant à des histiocytes.

\section{Discussion}

Initialement, il existait trois pathologies différentes : le granulome éosinophile[24], la maladie de Hand-schüller-Christian[27] et la maladie de Abt-Letterer-Siwe[22]. Le granulome éosinophile correspondait à une lyse osseuse localisée sans atteinte viscérale. La maladie de

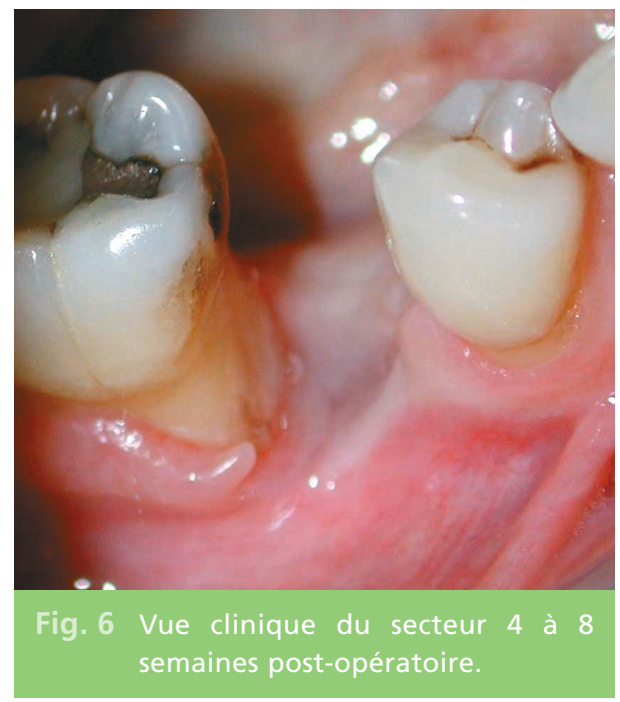

L'immunohistochimie pratiquée sur le prélèvement est très nettement positive aux anticorps $\mathrm{CD} 1 \mathrm{a}[17,25]$.

L'examen anatomopathologique conclut à la localisation gingivo-osseuse d'une histiocytose Langerhansienne[5].

\section{> Contrôles cliniques post-opératoires}

Un suivi clinique et radiologique a été mis en place. À 8 semaines post-opératoires (fig. 5 et 6), on peut constater que les sites d'intervention se sont ré-épithélialisés. L'examen radiographique ne montre pas de nouvelles zones de déminéralisation.

Les dents bordant les foyers d'histiocytose ne sont pas mobiles.

Hand-schüller-Christian est une maladie chronique décrite par des lésions osseuses, une exophtalmie et une polyurie. Finalement, la maladie de Abt-Letterer-Siwe aiguë est caractérisée par un rash cutané, une hépatomégalie, une splénomégalie, une anémie, une lym- 
phadénopathie et des lésions osseuses disséminées. En raison de leur analogie histologique, Lichtenstein[16] unifia ces trois entités sous le nom d'histicytose $X$. Nezelof et Basset[20] démontrèrent, quant à eux, que le $X$ correspondait à des cellules de Langerhans encore dénommées cellules dendritiques intraépithéliales.

Les présentations cliniques sont très hétérogènes. Les atteintes osseuses sont présentes dans $78 \%$ des cas. Un diabète insipide peut être rencontré dans près de $50 \%$ des cas. Comme pour notre patiente, les lésions maxillaires et mandibulaires sont fréquemment présentes et apparaissent au panoramique comme des images radio-claires uniques ou multiples. On décrit classiquement ces lésions par l'apparence de dents qui flottent dans le vide. Les atteintes cutanées sont rencontrées dans $50 \%$ des cas. Le foie et la rate peuvent être touchés. On retrouve des adénopathies dans $30 \%$ des cas.

Le diagnostic positif est posé par l'analyse anatomopathologique.

En raison de l'hétérogénéité des symptômes, Chu et coll.[5] ont proposé un arbre diagnostic :

- diagnostic préventif : image lacunaire radioclaire caractéristique ;

- diagnostic probable : image lacunaire radioclaire caractéristique associée à deux ou plus des signes suivants : coloration positive pour l'adénosine triphosphate, pour la protéine S-100, pour $\alpha$-D-mannosidase ;

- diagnostic de certitude: image lacunaire radio-claire caractéristique associée à la présence de granules de Birbeck dans les cellules de la lésion, ou une réaction positive pour l'antigène CD1a aux cellules de la lésion.

Une fois ce diagnostic de certitude établi, I'histiocytose Langerhansienne est alors caractéri- sée selon les signes cliniques. Egeler et coll.[10] ont proposé la classification suivante :

- histiocytose Langerhansienne non-extensive :

- lésions cutanées seules ou associées aux signes suivants :

- lésion mono-ostique avec ou sans diabète insipide, avec adénopathie ;

- lésions osseuses de plusieurs os ou plus de deux lésions sur un même os, avec ou sans diabète insipide, avec adénopathie.

- histiocytose Langerhansienne extensive :

- atteinte viscérale avec ou sans lésion osseuse, diabète insipide, adénopathie, mais sans signe de dysfonctionnement des poumons, du foie ou du système hématopoïétique :

- atteinte viscérale avec ou sans lésion osseuse, diabète insipide, adénopathie, mais avec signes de dysfonctionnement des poumons, du foie ou du système hématopoïétique.

Dans le cas que nous exposons, le diagnostic a été confirmé par de multiples biopsies et notamment une réaction positive aux CD1a des prélèvements buccaux. Par ailleurs, selon la classification de Egeler et coll.[10], il s'agit d'une forme non extensive puisqu'il n'y a pas d'atteinte viscérale.

La prise en charge des histiocytoses Langerhansiennes[19] dépend du type de lésion, du nombre de lésions, de leur accessibilité, du caractère récidivant et de l'existence d'atteintes viscérales.

Il est généralement admis que les foyers osseux isolés doivent être traités par un simple curetage chirurgical en première intention. Lorsqu'il existe plusieurs foyers ou lorsqu'une lésion récidive, les auteurs proposent différentes stratégies qui peuvent faire appel à la la chimiothérapie, aux traitements anti-inflam- 
matoires non-stéroïdiens per os, ou à l'injection intralésionelle de corticoïdes[19]. Certains auteurs ont également proposé de recourir à la radiothérapie à très faible dose des foyers.

Notre patiente a bénéficié d'un curetage des lésions crâniennes suivi de la mise en place de plaques d'ostéosynthèse, du curetage des lésions lombaires suivi d'une arthrodèse. Au niveau alvéolo-dentaire, conformément aux recommandations, nous avons procédé à l'avulsion des dents situées dans les territoires d'histiocytose et réalisé le curetage des foyers ainsi qu'une résection osseuse marginale. Les dents contiguës ont été laissées en place dans la mesure où elles ne présentaient aucune mobilité. Le pronostic est bon, le taux de récidive est de 7,3\%[3], mais la patiente doit être suivie de manière rigoureuse. Dans les premiers temps, il faut s'assurer de la ré-épithélialisation des alvéoles et contrôler cliniquement l'absence de récidive. Puis des panoramiques de contrôle seront effectuées tous les trois mois la première année puis tous les six mois. De façon conjointe, il a été mis en place un traitement anti-inflammatoire systémique de Cortancy $\mid{ }^{\circledR}$ à la dose de $60 \mathrm{mg}$ à raison de cures
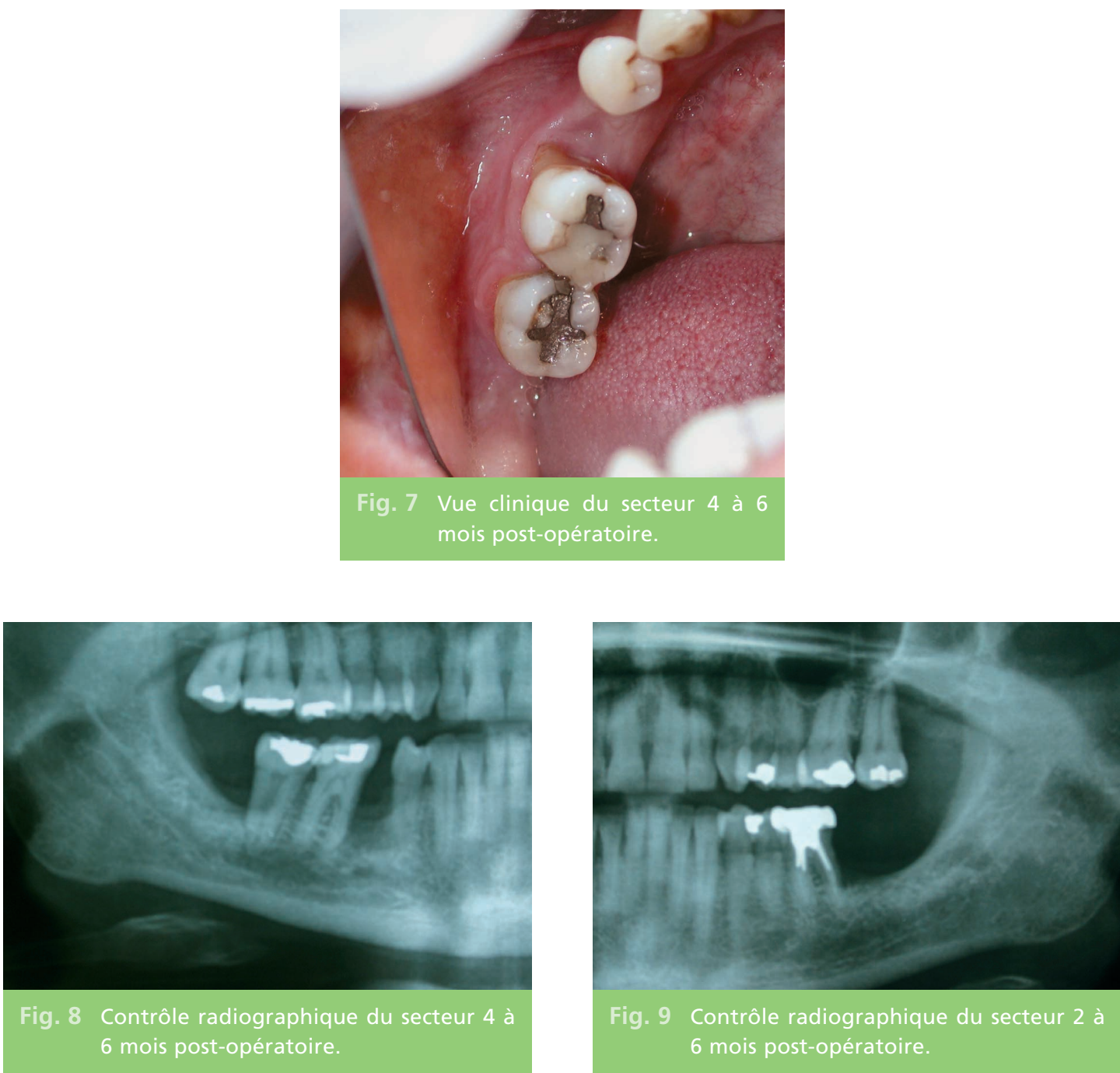

Fig. 9 Contrôle radiographique du secteur 2 à 6 mois post-opératoire. 


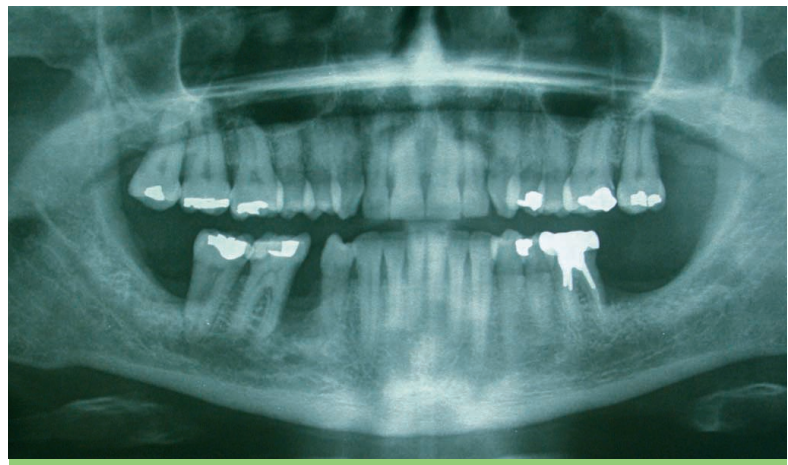

Fig. 10 Contrôle radiographique à 6 mois post-opératoire : absence de nouvelle localisation.

de 5 jours/semaine pendant 6 semaines consécutives et un traitement par Vinblastine à raison d'une cure par semaine pendant 6 semaines consécutives puis 1 cure toutes les 3 semaines, renouvelée 6 fois[3, 26].

À 6 mois post-opératoire, les sites d'intervention montrent une bonne cicatrisation

\section{Conclusion}

Les cas d'histiocytoses Langerhansiennes sont rares et d'aspects cliniques très divers. Le diagnostic est en général posé assez tardivement et la lésion initiale demeure difficile à identifier.

Les études, comme tend à le prouver notre cas, montrent que les lésions multifocales ne concernent pas que les enfants.

La plupart des histiocytoses Langerhansiennes

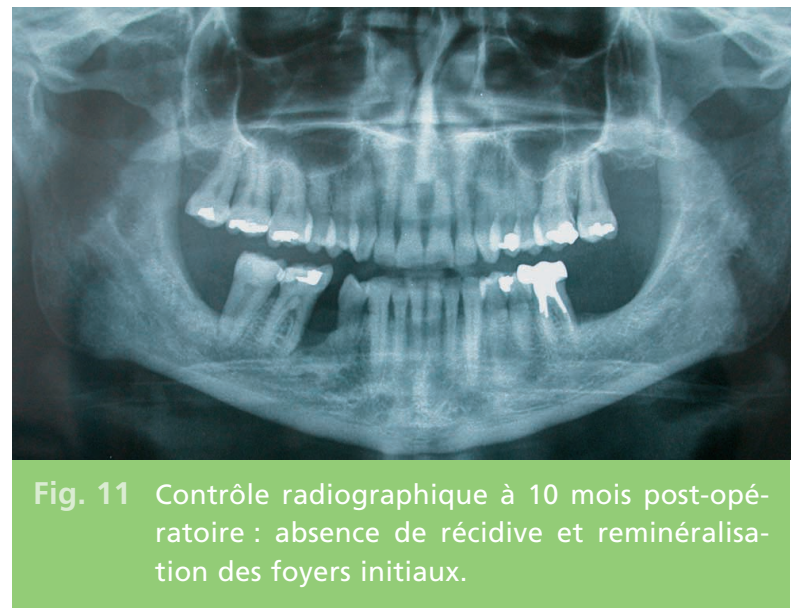

(fig. 7, 8 et 9) et on ne retrouve aucun foyer de récidive (fig. 10 et 11). Cependant, en tout état de cause, le suivi doit être maintenu à long terme pour deux raisons: s'assurer de l'absence de récidive, mais également pour prévenir l'apparition d'une nouvelle localisation.

ont une évolution bénigne. Les morbidités apparaissent surtout comme les conséquences des traitements systémiques administrés ou des traitements radiothérapiques.

Dans les atteintes multifocales, chaque patient doit être suivi cliniquement de manière stricte pendant au moins 1 an mais de façon plus générale les contrôles doivent se poursuivre tout le reste de la vie. 
Bibliographie

1. Histiocytosis syndromes

in children.

Writing Group

of the Histiocyte Society.

Lancet 1987;1(8526):208-209.

2. Arceci RJ, Brenner MK,

Pritchard J.

Controversies

and new approaches

to treatment of Langerhans

cell histiocytosis.

Hematol Oncol Clin North

Am 1998;12(2):339-357.

3. Ardekian L et al. Clinical and radiographic features of eosinophilic granuloma in the jaws: review of 41 lesions treated by surgery and low-dose radiotherapy.

Oral Surg Oral Med

Oral Pathol

Oral Radiol Endod

1999;87(2):238-242.

4. Burgener FA.

The radiological

manifestations

of disseminated

histiocytosis $\mathbf{X}$ in adults.

Rofo 1977;126(5):466-472.

5. Chu T et al.

Histiocytosis syndromes

in children.

Lancet 1987;2(8549):41-42.

6. Chu T, Jaffe R.

The normal Langerhans cell and the LCH cell.

Br J Cancer Suppl

1994;23:S4-10.

7. David R et al.

Radiologic features

of eosinophilic granuloma

of bone.

AJR Am J Roentgenol

1989;153(5):1021-1026.
8. Devaney KO, Putzi MJ,

Ferlito A, Rinaldo A.

Head and neck

Langerhans cell

histiocytosis.

Ann Otol Rhinol Laryngol

1997;106(6):526-532.

9. Eckardt A, Schultze A.

Maxillofacial manifestations

of Langerhans cell

histiocytosis:

a clinical and therapeutic

analysis of 10 patients.

Oral Oncol

2003;39(7):687-694.

10. Egeler RM et al.

The relation of

Langerhans cell histiocytosis

to acute leukemia,

lymphomas,

and other solid tumors.

The LCH-Malignancy Study

Group of the Histiocyte

Society.

Hematol Oncol Clin North

Am 1998;12(2):369-378.

11. Favara $B E$, Jaffe $R$.

Pathology of Langerhans

cell histiocytosis.

Hematol Oncol Clin North

Am 1987;1(1):75-97.

12. Green I, Behar AJ, Shanon E, Gorsky M.

Multifocal extraosseous

eosinophilic granuloma

of the head and neck.

Arch Otolaryngol Head Neck

Surg 1988;114(5):561-563.

13. Kaltsas GA et al.

Hypothalamo-pituitary

abnormalities

in adult patients

with Langerhans cell

histiocytosis: clinical,

endocrinological, and radiological features

and response to treatment.

$\mathrm{J}$ Clin Endocrinol Metab

2000;85(4):1370-1376.

14. Ladisch S, Gadner H.

Treatment of

Langerhans cell

histiocytosis-evolution

and current approaches.

$\mathrm{Br} J$ Cancer Suppl

1994;23:541-46.

15. Libicher $M$, Roeren $T$,

Troger J.

Localized Langerhans cell

histiocytosis of bone:

treatment and follow- up

in children.

Pediatr Radiol 1995;25

Suppl 1:S134-137.

16. Lichtenstein $\mathrm{L}$.

Histiocytosis $\mathrm{X}$;

integration of eosinophilic

granuloma of bone,

Letterer-Siwe disease,

and Schuller-Christian

disease as related

manifestations

of a single

nosologic entity.

AMA Arch Pathol

1953;56(1):84-102.

17. Lieberman PH et al.

Langerhans cell

(eosinophilic)

granulomatosis.

A clinicopathologic study

encompassing $\mathbf{5 0}$ years.

Am J Surg Pathol

1996;20(5):519-552.

18. McDonald JS, Miller RL, Bernstein $\mathrm{ML}$, Olson JW.

Histiocytosis $\mathrm{X}$ :

a clinical presentation.

J Oral Pathol

1980;9(6):342-349. 
19. Nakamura $S$, Bessho $K$, Nakao K, lizuka T, Scott RF. Langerhans' cell histiocytosis confined to the jaw.

J Oral Maxillofac Surg 2005;63(7):989-995.

20. Nezelof $C$, Basset F. From histiocytosis $X$ to Langerhans cell histiocytosis: a personal account. Int J Surg Pathol 2001;9(2):137-146.

21. Nicholson HS, Egeler RM, Nesbit ME. The epidemiology of Langerhans cell histiocytosis. Hematol Oncol Clin North Am 1998;12(2):379-384.

22. Nicolin LT.

Oral manifestations of the Abt-Letterer-Siwe disease.

Ann Stomatol (Roma) 1966;15(10):791-805.
23. Oelerich $M$, Vestring $T$. An unclear osteolysis of the right lower jaw. Histiocytosis X. Radiologe 1993;33(10): 597-599.

24. Otani S.
A discussion
on eosinophilic granuloma
of bone,
Letter-Siwe disease and Schuller-Christian disease.
J Mt Sinai Hosp N Y 1957;24(6):1079-1092.

25. Rosenfield NS, Abrahams J, Komp D.

Brain MR in patients with Langerhans cell histiocytosis:

findings and enhancement with Gd-DTPA.

Pediatr Radiol 1990;20(6):433-436.

26. Sellari-Franceschini $S$ et al. Langerhans' cells histiocytosis.

Int J Pediatr

Otorhinolaryngol

1999;48(1):83-87.

27. Sleeper EL.

Eosinophilic

granuloma

of bone;

its relationship

to Hand-Schuller-Christian

and Letterer-Siwe's

diseases

with emphasis

upon oral symptoms

and findings.

Oral Surg Oral Med

Oral Pathol

1951;4(7):896-918.

28. Steurer J, Furrer J,

Rhyner K, Vetter W,

Siegenthaler $\mathrm{W}$.

Histiocytosis X.

Clinical aspects

and course

in 5 patients.

Dtsch Med Wochenschr

1991;116(45):1702-1709.

\section{SUMMARY}

\section{Multifocal Langerhans cell histiocytosis.}

\section{A case report}

Fabrice CLIPET

Nora ALNO

Gérard LANCIEN

Gilbert DE MELLO

Keywords
- langerhans histiocytosis
- maxilla
- mandible
- curettage

Langerhans cell histiocytosis is a rare disorder characterized at the histological level by agregation of proliferating histiocytes.

The presented case concerns a Langerhans cell histiocytosis with multiple localization. Following the dental assessment, we notice the presence of clinical hurts being translated at the radiological level by radioclear images. The anatomopathological analysis which is positive to the CD1a confirms the Langerhans' etiology of this disease.

The treatment of the dental achievements consists of a surgical curettage in first intention. When an achievement recurs, diverse strategies can be then adopted: radiotherapy with low dose, chemotherapy, anti-inflammatory treatments.

In every case a very regular follow-up must be established. 


\section{Question - Réponse}

Au niveau bucco-dentaire, l'histiocytose Langerhansienne présente des signes analogues à une parodontite localisée.
Vrai
Faux

Ieı^ : әsuodə̧, 
Pour s'abonner, composer le :

tél. + 33 (0)1 $69181514-\mathrm{fax}+33(0) 169860678$

France et Union Européenne

version papier + version électronique $199 €$

Étudiant* : France et Union Européenne

(* sur présentation d'une attestation)

version papier + version électronique $119 €$

Reste du monde

version papier + version électronique $229 €$

Membre de I'AEOS

$179 €$

Prix au numéro

$65 €$

Frais d'envoi par avion

$49 €$

\section{NUMÉROS(*)}

(*) Offre valable jusqu'au 31/10/09

Réglé la somme de le

Chèque bancaire / postal $\mathrm{N}^{\circ}$

ou carte $\mathrm{N}^{\circ}$

(Partie à conserver)

(Partie à joindre à tout règlement)

$\square$ Je souhaite m'abonner pour

à la Revue AOS

$\square$ Je souhaite prolonger mon abonnement pour

à la Revue AOS

\section{MODE DE PAIEMENT :}

$\square$ Paiement effectué par chèque à l'ordre de : EDP Sciences - REVUE AOS

17 avenue du Hoggar - P.A. de Courtabœuf

B.P. 112 - 91944 Les Ulis Cedex A (France)

Tél. + 33 (0)1 69181514 - Fax + 33 (0)1 69860678

e-mail : abonnements@edpsciences.org

$\square$ Paiement par carte bancaire : $\square$ Carte Visa

Master Card

$\mathrm{N}^{\circ}$

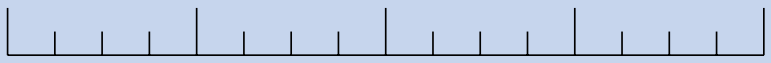

Noter ici les 3 derniers chiffres situés au dos de la carte

Date d'expiration $\lfloor|1|$

Signature obligatoire

\section{VOTRE ADRESSE :}

Vous êtes: $\square$ Chirurgien-Dentiste $\square$ Stomatologue $\square$ Orthodontiste $\square$ Autre: précisez

Nom :

Prénom :

Adresse :

Code Postal

Ville

Tél

Fax

E-mail 